\title{
Manejo ortopédico prequirúrgico de labio y paladar hendido unilateral: Reporte de casos con diferentes abordajes
}

\author{
Vilma Arteaga, ${ }^{1}$ Teresita Pannaci, ${ }^{2}$ Manuel Hinostroza, ${ }^{3}$ Giancarlo Brache. ${ }^{4}$
}

\begin{abstract}
Resumen: Pese a los avances de cirugía reconstructiva, el labio y paladara hendido unilateral (LPHU) representa un reto quirúrgico complejo cuando la brecha es mayor a $10 \mathrm{~mm}$. En países de recursos limitados, la distancia geográfica, falta de centros de referencia multidisciplinarios, recursos económicos de las familias y actualmente, la pandemia; limita el acceso de pacientes al tratamiento prequirúrgico. El presente estudio descriptivo reporta 2 casos de LPHU completo, de similar tamaño por inspección visual profesional, sexo masculino, no asociados a síndrome. El propósito del mismo es hacer una revisión de dos técnicas de ortopedia prequirúrgica: Placa de Ortopedia Funcional Maxilar (OFM)-Moldeado Nasal (MN); y el Retractor Nasal (RN)-Taping, como alternativa de tratamiento a distancia. El paciente con placa OFM-MN fue tratado en el 2015, presencial, el paciente con RNTaping, en el 2021 por telemedicina y un promotor de salud capacitado. Los resultados de simetría nasal y maxilar se midieron con software ImageJ, a través de 4 mediciones lineales y una angular, basadas en el método descrito por Barillas y de mediciones lineales realizadas a los modelos de yeso como las propuestas por Mazaheri. Las técnicas descritas para ambos pacientes lograron disminuir el ancho de la hendidura y moldear el cartílago alar del lado hendido. La técnica OFM-MN mostró ventajas en el desarrollo del arco maxilar promoviendo aumento de volumen de hueso y desarrollo en los tres planos del espacio sin colapso del arco. La técnica RN-Taping, restringió el arco maxilar, sin embargo, podría ser una alternativa de tratamiento a distancia.
\end{abstract}

Palabras clave: ortopedia, retractor nasal, ortopedia prequirúrgica.

\section{Tratamento ortopédico pré-cirúrgico de fissura labiopalatina unilateral: relato de casos com diferentes abordagens}

\begin{abstract}
Resumo: Apesar dos avanços na cirurgia reconstrutiva, a fenda labiopalatina unilateral (LPHU) representa um desafio cirúrgico complexo quando o gap é maior que $10 \mathrm{~mm}$. Em países com recursos limitados, distância geográfica, falta de centros de referência multidisciplinares, recursos econômicos das famílias e, atualmente, a pandemia; limita o acesso do paciente ao tratamento pré-cirúrgico. O presente estudo descritivo relata 2 casos de LPHU completa, de tamanho semelhante por inspeção visual profissional, do sexo masculino, não associada à síndrome. Seu objetivo é revisar duas técnicas ortopédicas pré-cirúrgicas: Placa Ortopédica Funcional Maxilar (OFM) - Moldagem Nasal (MN); e o Retrator Nasal (RN)-Taping, como alternativa de tratamento remoto. O paciente com placa OFM-MN foi atendido em 2015, pessoalmente, o paciente com RN-Taping, em 2021 por telemedicina e promotor de saúde treinado. Os resultados da simetria nasal e maxilar foram medidos com o software ImageJ, por meio de 4 medidas lineares e uma angular, com base no método descrito por Barillas e medidas lineares feitas nos modelos de gesso como os propostos por Mazaheri. As técnicas descritas para ambos os pacientes foram capazes de reduzir a largura da fenda e moldar a cartilagem alar do lado da fenda. A técnica OFM-MN apresentou vantagens no desenvolvimento do arco superior, promovendo aumento do volume ósseo e desenvolvimento nos três planos do espaço sem colapso do arco. A técnica RN-Taping restringiu a arcada superior, porém pode ser uma alternativa para tratamento remoto.
\end{abstract}

Palabra-chave: ortopedia funcional maxilar, afastador nasal, ortopedia pré-cirúrgica.

\footnotetext{
${ }^{1}$ DDS, Asociación Académica Guatemalteca de Odontopediatría, Guatemala.

${ }^{2}$ DDS, Colaborador Investigador Senior en el Instituto de Investigación VHIR Vall d 'Hebron, Barcelona, España.

${ }^{3}$ DDS, MSc, Perú.

${ }^{4}$ DDS, República Dominicana.
} 


\section{Presurgical orthopedic management of unilateral cleft lip and palate: Report of cases with different approaches}

Abstract: Despite the advances in reconstructive surgery, unilateral cleft lip and palate (UCLP) represents a complex surgical challenge when greater than $10 \mathrm{~mm}$. In low resource settings, distance, lack of multidisciplinary centers, low income and currently, the pandemic; limits patients to access to presurgical treatment. This study reports 2 cases of complete UCLP, similar in size by professional visual inspection, male, not associated with any syndromes. The purpose is to review two presurgical orthopedic techniques: Maxillary Functional Orthopedic Plate (MFO) -Nasal Molding (NM) and Nasal Retractor (NR) -Taping as a teledentistry alternative. The patient with the FMO-NM plate was treated in 2015, in person; the NR-Taping patient, in 2021, by telemedicine and a trained health promoter. The results of nasal and maxillary symmetry were measured with ImageJ software, through 4 linear measurements and one angular, based on the method described by Barillas and linear measurements made on the plaster models such as those proposed by Mazaheri. The techniques described for both patients were able to reduce the width of the cleft and mold the alar cartilage on the cleft side. The OFM-MN technique showed advantages in the development of the maxillary arch by guiding growth and development in the three planes of the space without collapse of the arch. The NR-Taping technique restricted the maxillary arch but is an alternative to remote treatment.

Keywords: maxillary functional orthopedics, nasal retractor, presurgical orthopedics.

\section{Introducción}

La ortopedia prequirúrgica aplicada a pacientes nacidos con labio y paladar hendido se inició a mediados del siglo pasado con la placa descrita por McNeil, en 1993 el Dr Barry Grayson intrudujo la técnica de Moldeado Naso-alveolar (NAM por sus siglas en inglés). En 2013 se intrudujo el concepto Dynacleft como un dispositivo ortopédico alternativo enfocado en el moldeado del cartílago alar por medio de fuerza de tracción. ${ }^{1}$

A pesar de los avances en cirugía reconstructiva, el labio y paladar hendido unilateral (LPHU) representa un reto quirúrgico complejo directamente proporcional al tamaño y tipo de hendidura. El objetivo principal del tratamiento en pacientes con LPHU es lograr una anatomía lo más normal y funcional posible.
De acuerdo al análisis de la situación de salud bucal de Guatemala en 2019, la red de servicio de salud pública cuenta con 1 odontólogo por cada 84000 habitantes cuya ubicación es inequitativa, lo que hace necesario la capacitación de promotores de salud que puedan llegar a la población rural más alejada ${ }^{2}$. La atención temprana se dificulta debido a que los centros de atención multidisciplinaria son escasos y muchas veces quedan distantes a los lugares de residencia del paciente; y las familias en muchos casos no tienen los recursos económicos suficientes para llevar a los pacientes con la frecuencia requerida a estos centros de referencia.

Múltiples reportes de caso evidencian los beneficios de la terapia ortopédica prequirúrgica permitiendo una cirugía menos invasiva y mejores resultados estéticos y funcionales. En la última década 
distintas técnicas han sido utilizadas por varios centros multidisciplinarios alrededor del mundo con este fin. ${ }^{3,4}$

El propósito común de la técnica de Ortopedia Funcional Maxilar (OFM)Moldeado Nasal (MN), descrita por Pannaci $^{5}$ y el Retractor nasal (RN)-Taping modificación Hinostroza ${ }^{6}$ en pacientes con LPHU es disminuir la amplitud de la brecha alveolar y palatina y moldear el cartílago alar del lado afectado para reducir la necesidad de disecciones mayores con la consecuente tensión en el labio superior ya reparado. La diferencia entra ambas técnicas es la placa OFM, que no se usa en la técnica RN-Taping.

La técnica prequirúrgica OFM-MN persigue conformar el arco maxilar en $U$ promoviendo el cierre de la brecha por crecimiento óseo en los segmentos alveolares y palatinos, moldear el cartílago alar del lado afectado para que adquiriera forma de domo, enderezar y elongar la columela, lograr proyección de punta nasal, promover el crecimiento vertical de ambos segmentos y desarrollar el tercio medio facial en los tres planos del espacio ${ }^{5}$. Por su parte, la técnica RN-Taping persigue aproximar los segmentos óseos y el tejido blando, moldear el cartílago alar del lado afectado en forma de domo, alinear, enderezar y elongar la columela y punta nasal. ${ }^{6}$

En 2020 la pandemia del Covid-19 limitó la atención prequirúrgica en consultorios, retrasando las cirugías y teniendo que ajustar los tratamientos al momento vivido, a través de consultas por telemedicina. Ante las restricciones que obligan las normas impuestas por la pandemia la opción al tratamiento es la técnica Retractor Nasal (RN)-Taping.
El objetivo de este estudio es describir y comparar dos casos de LPHU similares, ventajas y desventajas del manejo prequirúrgico con técnicas distintas, haciendo una revisión de los resultados obtenidos en 4 meses de tratamiento.

\section{Materiales y métodos}

La terapia prequirúrgica debe iniciar idealmente al momento del nacimiento ${ }^{3-5,9}$. En países de recursos limitados, la atención temprana y oportuna se dificulta debido a que los centros de referencia para la atención multidisciplinaria de estos pacientes son escasos. Este estudio es un reporte de dos casos con fines descriptivos. Los casos fueron seleccionados por su similitud de acuerdo a observación profesional. Ambos casos son masculinos, nacidos con LPHU completo derecho, en quienes se inicia la terapia a los 2 meses de edad. Se obtuvo consentimiento informado de los padres y todos los datos registrados se realizan sin identificadores personales.

La terapia OFM-MN ${ }^{5,12,13}$ consiste en la colocación de una placa acrílica, de preferencia al momento del nacimiento, con el fin de aumentar el volumen y dirigir el crecimiento de los segmentos maxilares por medio de estimulación de la musculatura lengua-oro-facial, alineando el arco maxilar. La placa separa la cavidad oral de la cavidad nasal permitiendo la respiración nasal y el adecuando apoyo de la lengua, mejorando la capacidad de succión y promoviendo una rehabilitación funcional. Debe cambiarse cada 4 semanas y utilizarse 24 horas al día. Al disminuir la hendidura alveolar a $5 \mathrm{~mm}$ o menos se 
añade el dispositivo nasal, conformado con alambre de acero 0,7 mm (Dynaflex ${ }^{\circledR}$ ) en forma de cuello de cisne con doblez en forma de riñon recubierto de acrílico rígido $\left(\right.$ NicTone $^{\circledR}$ ) y acrílico suave (Durabase ${ }^{\circledR}$ ). Los controles del dispositivo nasal consisten en añadir en cada cita acrilico suave (Durabase ${ }^{\circledR}$ ) para compensar el cambio de forma del cartílago y mantener la misma presión inicial. $^{5}$

La terapia con RN-Taping consiste en la colocación de un retractor nasal confeccionado en alambre de acero $0,6 \mathrm{~mm}$ (Dynaflex ${ }^{\circledR}$ ); 6 dobleces con alicate 139 $\left(\mathrm{Ormco}^{\circledR}\right.$ ) y protector de tubo (DLL ${ }^{\circledR}$ ), medido desde punta de nariz a línea intercantal interna de los ojos y colocado traccionando hacia la frente por medio de micropore $\left(3 \mathrm{M}^{\circledR}\right)$. El retractor se coloca paralelo a la línea media facial. Después de 30 días de uso en esta posición el brazo largo del $\mathrm{RN}$ debe posicionarse de manera inclinada hacia lado no afectado cuidando de no bloquear la visión del ojo, y continuar fijándolo a la piel de la frente con adhesivo micropore; esta posición se debe mantener hasta el día de la cirugía. La fuerza de tracción del RN es de 1 onza medida con un dinamómetro, en el caso de no contar con un dinamómetro se indica, una vez colocado en el ala nasal afectada, realizar la tracción del RN hasta llegar a la altura del cartílago del lado no afectado. Al mismo tiempo se indica el uso de taping (cinta elástica adhesiva Theraband $K$ tape $^{\circledR}$ ) adherido primero del lado no afectado y desde la linea nasogeniana, traccionando el segmento del labio del lado afectado y extendiendose hasta la altura del canto externo del ojo del lado afectado. La cinta elástica adhesiva debe removerse cada 2 a 4 días para evitar lesionar la piel. ${ }^{6}$

\section{Caso 1 Técnica prequirúrgica OFM-MN}

Paciente de sexo masculino, nacido con LPHU completo derecho en 2015, en el área urbana de la ciudad Capital de Guatemala; llegó a una consulta privada en clínica Ortodent, referido por la clínica privada Nugenesis Plastic Surgery.

Basado en la filosofía de Ortopedia Funcional de los Maxilares y las teorías de crecimiento óseo de Moss, Matriz Funcional y osteosíntesis generada por fuerzas intermitentes, luego de la evaluación clínica, se decidió comenzar con preparación prequirúrgica con la técnica OFM-MN. Se tomó una impresión con alginato (Emulate ${ }^{\circledR}$ ) utilizando para ello una cubeta individual de acrílico (Triad, Dentsplay Sirona ${ }^{\circledR}$ ), agregándole cera de utilidad (MDC Dental ${ }^{\circledR}$ ) en los bordes, cubierta por algodón, para mejorar la adhesión del material. Se obtuvo un modelo de estudio y trabajo de yeso piedra tipo III, el cual fue analizado y trabajado, encerando la zona palatina y alveolar que involucra el defecto óseo para promover crecimiento de hueso y realizando un desgaste cuidadoso en el yeso (raspado) en los lugares donde se requería generar más estímulo por el mayor contacto de la placa con la mucosa, logrando así umentar el volumen de los segmentos con la consecuente disminución del ancho de la hendidura en toda su extensión.

Siguiendo el protocolo, la placa OFM se cambió cada 4 semanas, se tomó impresión y se evaluaron los modelos con mediciones basadas en las propuestas por Mazaheri ${ }^{10}$. De acuerdo a las mediciones lineales del primer modelo de estudio, la hendidura alveolar era de $11 \mathrm{~mm}$. Al tercer mes de tratamiento la hendidura alveolar disminuyó 

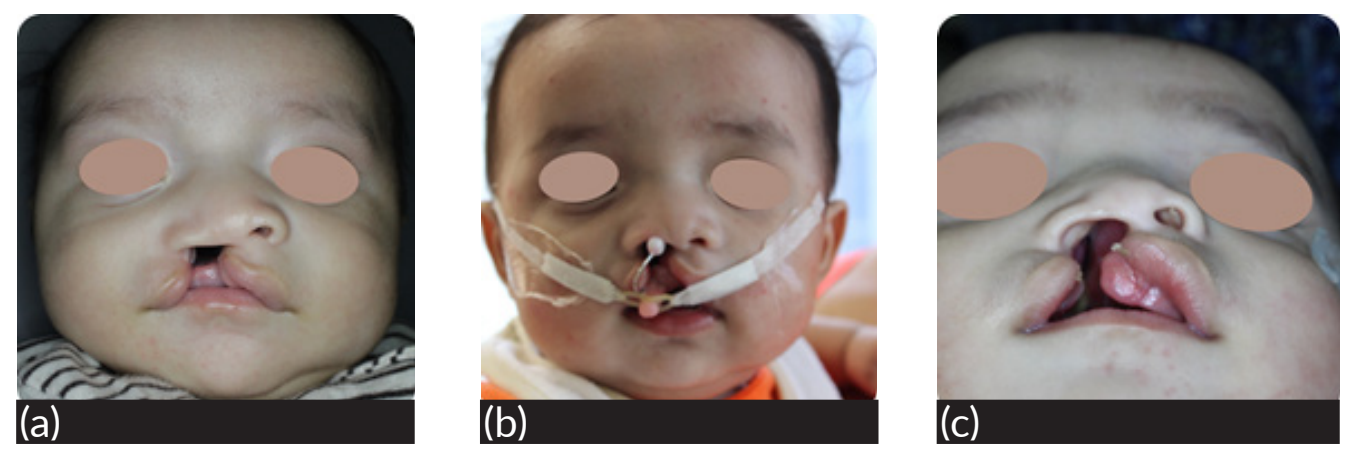

Figura 1. Fotos Ortodent-V.A. (a) inicial, (b) tratamiento prequirúrgico placa Ortopedia funcional maxilar-moldeado nasal, (c) vista basal a los 4 meses de tratamiento.

un total de $6 \mathrm{~mm}$ por crecimiento óseo y aumento de volumen de los segmentos maxilares, siendo la medida líneal de la hendidura de $5 \mathrm{~mm}$. Se adhiere a la placa OFM el dispositivo nasal en esta etapa. El dispositivo nasal ejerce una presión constante al cartílago, la dirección de la fuerza es hacia arriba, hacia adelante y convergente a la línea media. El tiempo total del tratamiento prequrúrgico fue 6 meses, momento en que la hendidura alveolar tenía $3 \mathrm{~mm}$ de amplitud. La queiloplastía y rinoplastía primaria se realizaron a los 8 meses de edad (Figura 1).

\section{Caso 2 Técnica prequirúrgica RN-Taping}

Paciente de sexo masculino, nacido con LPHU completo derecho en enero 2021, con residencia en área rural. Referido por la promotora de salud del área de Jalapa,

a través de Asociación Compañero para
Cirugía (ACPC). Se recibió la información de la medida de la altura nasal con lo que se confeccionó el RN en la clínica Ortodent en la ciudad capital y una vez elaborado se envió por medio de ACPC hasta la ciudad donde reside el paciente. La promotora instruyó a la mamá la forma correcta de colocar el RN. Se indicó introducir la parte activa (en forma de gancho) en el ala nasal del lado afectado cuidando de traccionarlo por el brazo largo del RN hacia la frente, donde debe fijarse a la piel con adhesivo micropore.

Al mismo tiempo se indica el uso de taping como lo indica la técnica. El tiempo total del tratamiento prequirúrgico fue de 4 meses. La queiloplastía y rinoplastía primaria se realizaron en el paciente a los 6 mesed de edad, momento en el cual se toma una impresión de alginato (Emulate ${ }^{\circledR}$ ) y se obtiene un modelo de estudio en yeso tipo III (Figura 2).
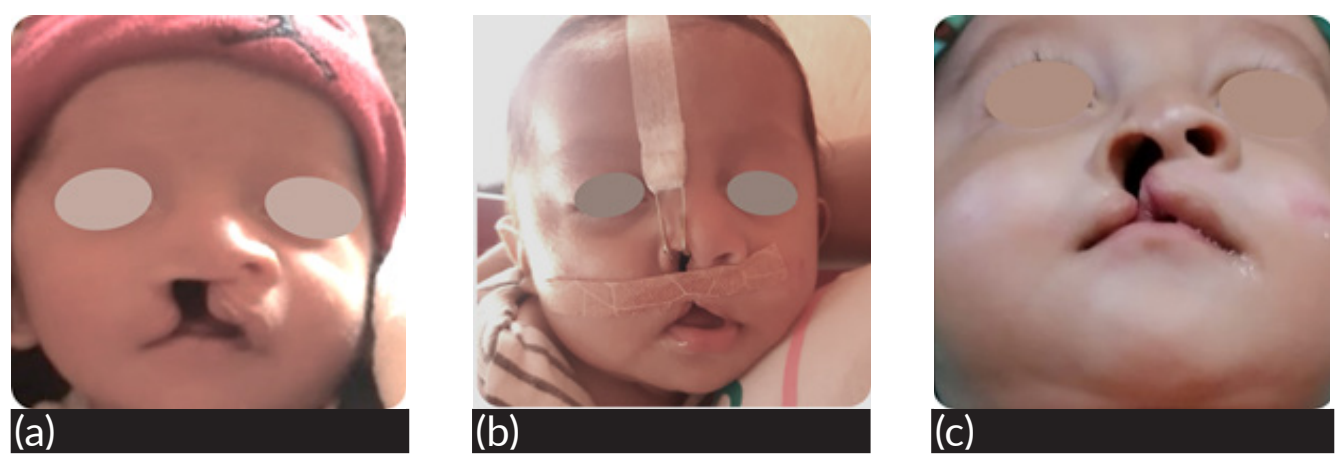

Figura 2. Fotos ACPC-V.A. (a) Inicial (b)tratamiento Retractor nasal-taping (c) resultados vista basal. 


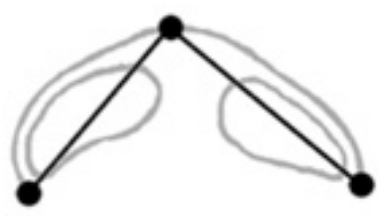

a) NADPL

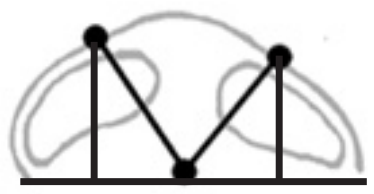

b) $\mathrm{NDH} / \mathrm{NDH}-\mathrm{B}$

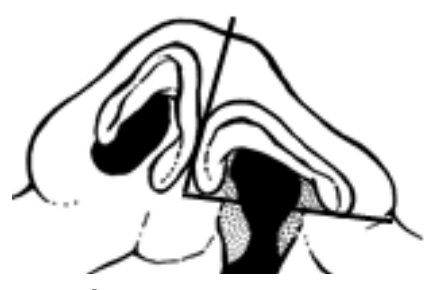

c) Ángulo de Columela

Figura 3. Medidas antropométricas: (a) NADPL, proyección lineal del domo alar; (b) NDH, altura del domo alar (Barillas et al 2009) y NDH-B altura del domo alar respecto a la base nasal propuesta en este estudio (c) el ángulo de la columela, de la base de la narina afectada a la línea de longitud de la columela propuesto en este estudio 7,8

\section{Resultados}

Para la toma de medidas angulares y lineales en modelos y fotografías se utilizó el software ImageJ. Las medidas fueron tomadas por el mismo investigador, 3 veces en días consecutivos distintos. Se utilizó el promedio de las medidas para el análisis.

\section{Simetría Nasal}

La simetría nasal se evaluó de acuerdo a 4 mediciones lineales, a saber, proyección lineal del domo nasal (NADPL por sus siglas en inglés), altura del domo nasal (NDH) respecto a la columela, como descrito por Barillas et al. ${ }^{7}$ El presente artículo propone 3 medidas adicionales, la altura del domo respecto a la base nasal (NDH-B), el ángulo de desviación de la columela y la longitud de la misma (Figura 3). De las medidas lineales, se obtuvo el ratio del lado no afectado con respecto al lado afectado de las 3 primeras medidas; se comparó la longitud de la columela, tomada de la base a la punta de la nariz, además, se tomó una medida angular de corrección de la columela con respecto a la línea base de la narina del lado afectado, trazada del canto externo del cartílago alar al punto medio de la base de la columela. La ratio del ángulo de la columela para cada caso se obtuvo respecto a la medida normal de $90^{\circ}$.

Los resultados obtenidos demuestran que el porcentaje de simetría nasal de acuerdo a las 3 primeras medidas es más favorable para el paciente tratado con RN-Taping. La corrección de la desviación de la columela fue más favorable en el paciente tratado con la técnica OFM-MN (Tabla 1, Gráfico 1).

Tabla 1. Porcentaje de simetría nasal de técnicas diferentes, utilizadas en 2 pacientes con fisuras similares y diferente acceso a servicios de salud, a los 6 meses de edad.

\begin{tabular}{lcccc}
\hline \multirow{2}{*}{ Tratamiento prequirúrgico } & \multicolumn{4}{c}{ Porcentaje de Simetría Nasal a los 4 meses de tratamiento } \\
\cline { 2 - 5 } & $*$ NADPL (\%) & †NDH & ‡NDH-B & Desviación de Columela \\
\hline Ortopedia funcional maxilar-moldeado nasal & 84.478 & 60.193 & 81.953 & 91.156 \\
Retractor nasal-taping & 97.801 & 62.929 & 101.385 & 82.059 \\
\hline
\end{tabular}

*NADPL, proyección lineal del domo alar

†NDH, altura del domo alar

$\ddagger N D H-B$, altura del domo alar con respecto a la base 
PORCENTAJE DE SIMETRÍA NASAL

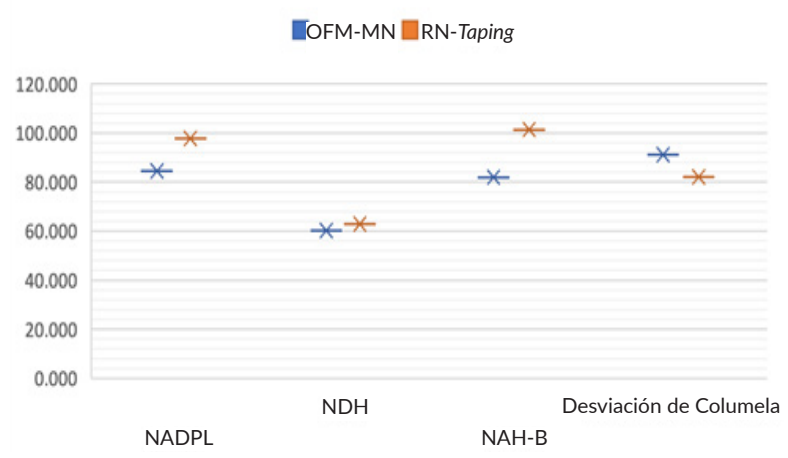

Grafíco 1. Simetría nasal obtenida con técnicas prequirúrgicas diferentes, utilizadas en 2 pacientes con fisuras similares y diferente acceso a servicios de salud, a los 6 meses de edad.

Los parámetros de longitud de la columela en ambas técnicas se encuentran dentro de la norma, sin embargo la longitud de la columela es mayor en el paciente tratado con RN-Taping ${ }^{11}$ (Gráfico 2).

\section{Conformación del arco maxilar}

En ambas técnicas se evidencia aproximación de los segmentos maxilares. Los resultados que se presentan son las medidas en modelos de estudio, basadas en las publicadas por Mazaheri (Figura 4) ${ }^{10}$. Ambos modelos tomados a los 6 meses de edad, cuando se habían cumplido 4 meses de tratamiento (Figuras 5 y 6 ).
LARGO DE COLUMELA

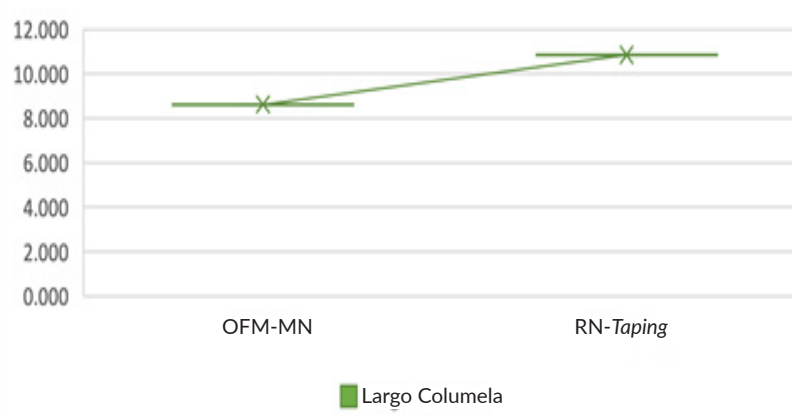

Grafíco 2. Largo de la columela a los 4 meses de tratamiento. El rango normal es de 5 a $9 \mathrm{~mm} .{ }^{10}$

En el paciente tratado con OFM-MN, el maxilar en el plano transverso tuvo mayor desarrollo que en el paciente tratado con RN-Taping. El desarrollo del largo del arco fue mayor en el paciente tratado con placa OFM-NAM. La forma de arco en el paciente tratado sin placa OFM es asimétrica, mientras que la forma del arco en el paciente tratado con placa OFM muestra simetría. En el plano sagital hay mayor diferencia entre el segmento menor y el mayor en el paciente que solo usó RN-Taping sin placa OFM. En el paciente tratado con placa OFM-MN se observa mayor desarrollo en el plano sagital. En el paciente que solo usó $\mathrm{RN}$-Taping se evidencia cierre de hendidura por colapso maxilar. Adicionalmente se observa desviación hacia la línea media de los tercios anterior y medio del segmento

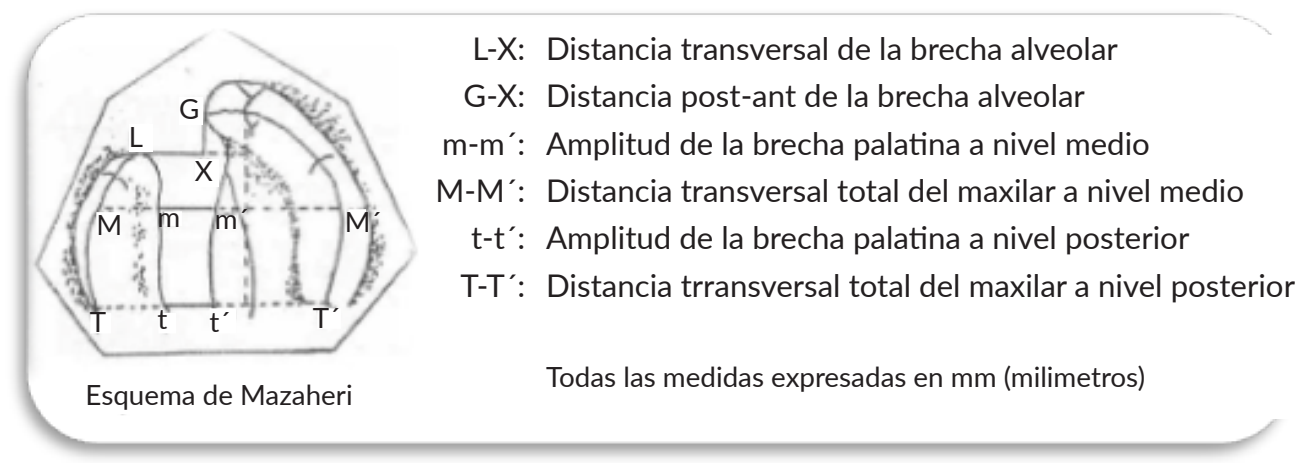

Figura 4. Medidas lineales realizadas para evaluar el desarrollo del maxilar en ambos pacientes. Esquema de medidas Mazaheri. ${ }^{10,11}$ 
Tabla 2. Medidias lineales $(\mathrm{mm})$ de desarrollo del arco maxilar en 2 pacientes tratados por diferente técnica prequirúrgica durante cuatro meses.

\begin{tabular}{lccccccc}
\hline & Ancho del maxilar & \multicolumn{2}{c}{ Longitud de arco } & \multicolumn{2}{c}{ Hendidura alveolar } & \multicolumn{2}{c}{ Hendidura palatina } \\
\cline { 2 - 8 } Tratamiento & T-T' $^{\prime}$ & M-M' & I-T-T' & L-X & G-X & m-m & t-t' \\
\hline OFM-MN & 47.784 & 46.128 & 25.710 & 7.352 & 3.524 & 15.507 & 18.504 \\
RN-Taping & 44.769 & 40.665 & 23.362 & 4.208 & 4.620 & 13.796 & 16.954 \\
\hline
\end{tabular}

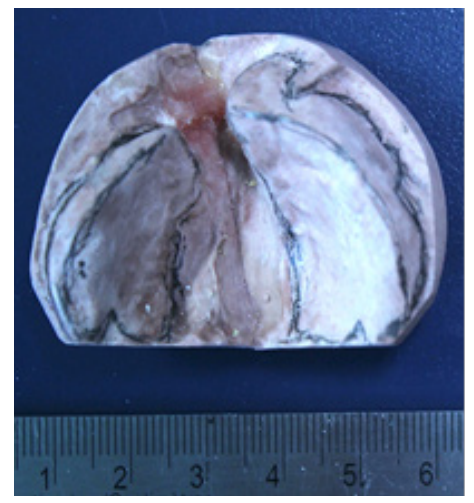

Figura 5. Caso 1 OFM-MN.

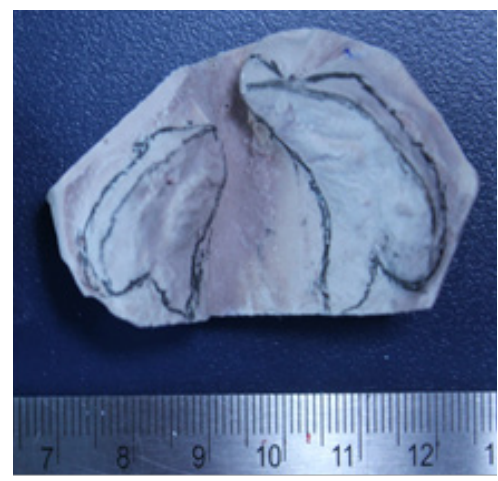

Figura 6. Caso $2 \mathrm{RN}$-Taping

menor por lo que no se encuentra bien relacionado con respecto al segmento mayor, persistiendo el colapso en el plano transverso. Al ver las fotos de la Figura 5 y 6 de los modelos del maxilar superior se observa que solo en la porción terminal de los tercios anteriores de ambos segmentos mayor y menor, hubo un mayor cierre del ancho de la hendidura en el paciente con RN-Taping. Sin embargo, la diferencia en ancho de la hendidura palatina entre los

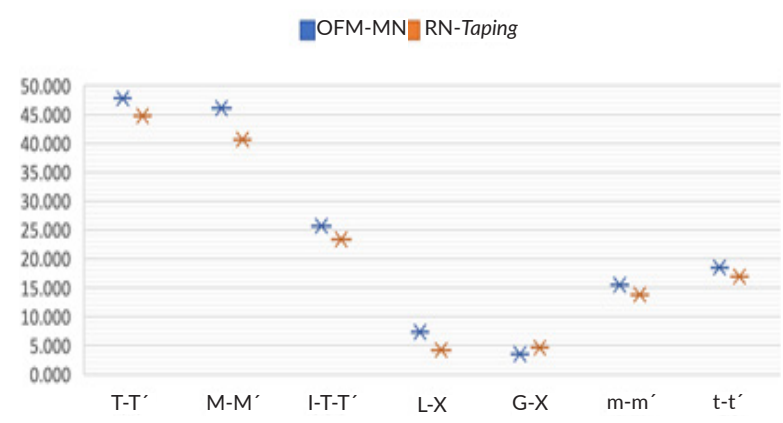

Grafíco 3. Grafico que representa los cambios de crecimiento y desarrollo del maxilar superior en ambos casos respecto al ancho, longitud de arco y amplitud de brecha alveolar y palatina.

tercios medios y sobre todo posteriores entre los segmentos no es significativa al compararlo con el paciente que usó OFMMN. (Tabla 2, Gráfico 3).

\section{Discusión}

Las técnicas descritas para ambos pacientes lograron disminuir el ancho de la hendidura alveolar y moldear el cartílago alar del lado hendido a una forma de domo evidenciándose mejores resultados estéticos posterior a la queiloplastía y rinoplastía primaria.

El caso tratado con OFM-MN permitió redireccionar ambos segmentos maxilares en forma de "U", promoviendo el crecimiento 
óseo, lo que le proporciona mejor soporte a los tejidos blandos al momento de la queiloplastía y mayor desarrollo del tercio medio facial en los tres planos del espacio. A largo plazo esta técnica promueve una mejor oclusión evitando mordidas cruzadas unilaterales ocasionadas por el colapso en el plano transverso. La mejora en el ángulo de la columela es consecuencia del desarrollo anteroposterior del segmento menor, que hace que la línea de la base nasal sea más horizonal. Entre las desventajas de la técnica de OFM-MN se encuentran: a) Se requieren controles periódicos presenciales cada cuatro (4) semanas, para cambiar la placa y activar el dispositivo nasal. b) Se requiere mayor tiempo de trabajo de laboratorio y mayor cantidad de material utilizado, lo que aumenta el costo de dicho tratamiento, sin embargo, proporciona mejores resultados a largo plazo.

La técnica RN-Taping es una opción de tratamiento que puede ser usada en países con recursos limitados, en comunidades vulnerables donde no hay acceso a servicios de salud con atención multidisciplinaria, dificultándoseeldesplazamientodelpaciente y sus padres por la distancia geográfica y los problemas económicos que enfrentan las familias. Se puede realizar y monitorear a distancia a través de la telemedicina en los casos de pacientes donde su lugar de residencia sea en una zona rural o distante del centro de salud y su costo económico es notablemente menor que el anterior. Entre las desventajas de la técnica RN-Taping se encuentran: a) la fuerza mecánica utilizada para el cierre de la hendidura a través del taping no produce impacto positivo en el crecimiento y desarrollo del maxilar que pudo observarse en el caso 2, lo que traerá como consecuencia hipoplasia del tercio medio facial y mordida cruzada unilateral del lado afectado, y afectará las funciones de respiración, deglución y masticación. b) La tracción excesiva del cartílago alar del lado afectado produjo en este caso el adelgazamiento del mismo, pudiendo producir dificultades al momento de realizar la rinoplastia primaria.

\section{Conclusiones}

Las técnicas ortopédicas prequirúrgicas presentadas en este trabajo han sido de utilidad para mejorar los resultados estéticos de tejidos blandos y evitar cirugías extensas y tensión excesivas durante la cicatrización.

El estudio individualizado del caso, donde se valoren las necesidades de tratamiento del paciente, el nivel de compromiso y las posibilidades económicas familiares, el acceso de servicios de salud con atención multidisciplinaria en sus sitios de residencia para asistir a sus controles sucesivos, entre otros aspectos; serán determinantes en la toma de decisiones del caso.

Ambos tratamientos muestran beneficio en la conformación y moldeado del cartílago alar y en el cierre del ancho de la hendidura. Sin embargo, los mejores resultados para el paciente se obtienen del tratamiento con OFM-MN.

Entre las ventajas de la OFM-MN, la placa OFM proporciona mejores resultados en cuanto a la conformación del arco maxilar y crecimiento del tercio medio facial en los 3 planos del espacio al comparar con la técnica RN-Taping. 
Entre las ventajas del tratamiento $\mathrm{RN}$ Taping se mencionan que es económico y puede realizarse a través de promotores de salud guiados por un profesional a distancia. Proporciona menores retos para los padres o cuidadores. Sin embargo, como desventaja se encuentra que no promueve el crecimiento de los segmentos maxilares.

\section{Recomendaciones}

Se sugiere estudiar la combinación de las técnicas de la placa de OFM y el RN a futuro utilizando las medidas de simetría propuestas en este estudio.

Los padres o cuidadores deben ser entrenados sobre el uso correcto del RN para evitar que se adelgace el cartílago alar del lado afectado o que su longitud final sea excesiva.

Se recomienda realizar estudios con mayor número de casos.

\section{Referencias bibliográficas}

1. Smith K, Henry B, Scott M. Presurgical Dentofacial Orthopedic Management of the Cleft Patient. Oral Maxillofac Surg Clin North Am. 2016;28(2):169-176.

2. Vanegas C, Saenz W, González M, Perez D, Villagran E, Sánchez E et al. Análisis de la Situación de Salud Bucal en Guatemala. Guatemala: Comisión Nacional de Salud Bucal (CONASABU); 2018 p. 13-14.

3. Gibson, E., Pfeifauf, K., Skolnick, G., Kim, A., Naidoo, S., Snyder-Warwick, A., Huebener, D. and Patel, K. Presurgical Orthopedic Intervention Prior to Cleft Lip and Palate Repair: Nasoalveolar Molding Versus Passive Molding Appliance Therapy. J. Craniofacial. Surg. 2020; 32(2): 486-491.

4. Santiago, P., and Grayson, B. Role of the Craniofacial Orthodontist on the Craniofacial and Cleft Lip and Palate Team. Semin Orthod, 2009;15(4): 225-243.

5. Pannaci T, Arteaga V, Agell-Sogbe A, Kassam S, Scott M. Cleft Dental treatment, and Naso-Alveolar Molding. In: Swanson J, Low D, ed. by. Global cleft care in low-resource settings. 1st ed. Cham, Switzerland: Springer Nature; 2021: 353-375

6. Hinostroza-Flores M, Perona-Miguel de Priego G, Loo-Valle J. Tratamiento ortopédico prequirúrgico con retractor nasal modificación Hinostroza en fisura labiopalatina unilateral completa: Reporte de caso. Rev Cient. Odontol. (Lima). 2021; 9 (2): e065DOI: 10.21142/2523-2754-0902-2021-

7. Barillas I, Dec W, Warren S, Cutting C, Grayson B. Nasoalveolar Molding Improves Long-Term Nasal Symmetry in Complete Unilateral Cleft Lip-Cleft Palate Patients. Plast. Reconstr. 2009;123(3):1002-1006.

8. Henry, C., Samson, T. and Mackay, D. Evidence-Based Medicine. Plast. Reconstr. 2014;133(5):1276-1288.

9. Vinson, L. The Effect of DynaCleft ${ }^{\circledR}$ on Cleft Width in Unilateral Cleft Lip and Palate Patients. Int. J. Clin. Pediatr. Dent. 2017;41(6):42-445.

10. Mazaheri, M., Harding, R., Cooper, J., Meier, J. and Jones, T. Changes in arch form and dimensions of cleft patients. Am. J. Orthod. 1971;60(1):19-32.

11. Gamboam Criado Y. Antropometría general y craneofacial en neonatos. Bogotá DC Colombia: Universidad Nacional de Colombia Facultad de Odontología, Departamento de Salud Oral Posgrado Estomatología Pediátricay Ortopedia Maxilar; 2016 p. 34. Disponible en: https://fdocuments.net/document/antropometrageneral-y-craneofacial-en-neonatos-caso-bogot-2016-06-22-antropometra.html

Recibido: $14 / 11 / 21$

Aceptado: 22/01/22

Correspondencia: Vilma Arteaga, correo: arteagavilma2@gmail.com 